\title{
Application of Transversus Thoracis Muscle Plane Plus Rectus Sheath Block in the Perioperative Pain Management of Cardiac Surgery凶Study Protocol of a Randomized Double-blind Controlled Trial
}

Jierong Luo

Guangzhou First People's Hospital

Chengxiang Lu

Guangzhou First People's Hospital

Haitao Zhou

Guangzhou First People's Hospital

Guokun Ou

Guangzhou First People's Hospital

Lixin Xu

Guangzhou First People's Hospital

Bin Zheng ( $\nabla$ eyzhengbin@scut.edu.cn )

https://orcid.org/0000-0002-6580-8233

Study protocol

Keywords: transverse thoracic muscle plane block, recluse sheath block, cardiac surgery, perioperative pain management, analgesic dosage

Posted Date: June 28th, 2021

DOl: https://doi.org/10.21203/rs.3.rs-472782/v1

License: (c) (1) This work is licensed under a Creative Commons Attribution 4.0 International License. Read Full License 


\section{Abstract}

\section{Background}

Traditional cardiac surgical anesthesia is characterized by the use of large doses of opioids, accompanied by side effects such as oversedation, respiratory depression, long ventilator duration, prolonged ICU stay, opioid tolerance, addiction, and postoperative delirium. Fast track cardiac anesthesia under the Enhanced Recovery After Surgery (ERAS) concept requires quick recovery, rapid extubation, reduced perioperative opioid dosage and shorter ICU stay. An emerging regional block technique, transverse thoracic muscle plane block (TTPB) covers the Thoracic 2-6 (T2-T6) intercostal nerves and recluse sheath block (RSB), which can effectively relieve perioperative pain during median sternotomy. Bilateral TTPB plus RSB is expected to be a new analgesic mode in perioperative thoracotomy.

Methods

This is a single-center, randomized, double-blind, parallel controlled clinical trial. Eighty patients planning to undergo coronary artery bypass grafting or heart valve surgery via median sternotomy were randomly assigned 1:1 to the experimental group or control group. After general anesthesia, all subjects were injected with $0.3 \%$ ropivacaine (experimental group) or $0.9 \%$ normal saline (control group) $15 \mathrm{ml}$ (TTPB)and $10 \mathrm{ml}$ (RSB) on each side after insertion guided by B-ultrasound by anesthesiologist. The main outcomes were the threshold of incision pain and the total amount of analgesics used during the operation and 48h after the operation. Secondary outcomes were as follows: postoperative VAS score, duration of mechanical ventilation, length of stay in ICU, and hospitalization cost. In addition, random assignment is a computer program that generates random results. Patients, operators, and evaluators were grouped in a blind manner.

\section{Discussion}

The TTPB plus RSB on transthoracic median incision heart surgery is just a ramification of nerve block anesthesia. Thanks to the visualization development of ultrasound, the safety factor of TTPB and RSB is greatly improved and the block effect is more accurate, which provides a guarantee for the application. This study will provide evidence-based medical evidence and clinical data support for the application of TTPB and RSB in cardiac surgery.

Trial registration

ClinicalTrials.gov, NCT04838132. Registered 8 April 2021.Verson1.2. https://clinicaltrials.gov/ct2/show/NCT04838132?term=NCT04838132\&draw=2\&rank=1

\section{Background}

The "China Cardiovascular Disease Report 2018" pointed out that the prevalence and mortality of cardiovascular disease in China are still on the rise. It is estimated that the number of cardiovascular 
disease patients is 290 million, and the death rate of cardiovascular disease accounts for more than $40 \%$ of the residents' disease deaths[1]. Every year, more than 1.5 million patients undergoing cardiac surgery in the world face a series of factors that cause acute postoperative pain. The pain is related to hemodynamic instability, respiratory complications, psychological disorders $[2,3]$ and the risk of death afterwards [4]. Good postoperative analgesia after cardiac surgery can help patients recover quickly, get up early and shorten the length of hospital stay. At present, non-steroidal anti-inflammatory drugs and opioids are routinely used in clinical treatment of postoperative pain in patients undergoing cardiac surgery. However, the analgesic effect of opioids is not always sufficient and correspondingly brings many disadvantages, including lethargy, excessive sedation, respiratory depression, constipation, etc., which often lead to delayed recovery $[5,6]$. Modern medicine is in the era of rapid rehabilitation therapy, which emphasizes precision and individualized medical treatment, accompany with continuous updating and development of medical technology. Through reasonable perioperative management and a variety of methods, reduce the amount of opioids in the perioperative period, achieve rapid postoperative recovery, rapid extubation, and ultimately achieve safe and rapid recovery, greatly shorten the hospital stay, and save medical expenses [7]. Therefore, it is particularly important to develop a new type of analgesia model that is safe, effective and has fewer side effects for cardiac surgery with a median sternal incision.

Besides, remifentanil has quick effect, fast metabolism and strong analgesic effect, but it is easy to induce hyperalgesia and cause explosive pain. Study reported that the incidence of remifentanil hyperalgesia was $32.7 \%$ in patients with surgery duration of more than 2 hours, and $41.8 \%$ in patients with cumulative infusion of more than $30 \mu \mathrm{g} / \mathrm{kg}$. Intraoperative clinical infusion of $0.2 \mu \mathrm{g} / \mathrm{kg} / \mathrm{min}$ of remifentanil resulted in a reduction of the mechanical pain threshold in the area around the incision, increasing patients' demand for postoperative analgesics and causing hyperalgesia [8]. In the anesthesia management, there is an urgent need to find an ideal perioperative period management strategy, not only to make the anesthetic quickly effective, rapid metabolism, so as to quickly wake up the patient, but also to maintain the stability of hemodynamics and myocardial oxygen supply and demand balance, to avoid myocardial ischemia and even the occurrence of acute myocardial infarction, but also to reduce the application of opioids, to avoid pain sensitivity.

Transverse thoracic plane block is an emerging regional anesthesia technique that has analgesic effect on the anterior chest wall. TTPB was first reported by Ueshima et al. in 2015 [9]. TTPB is a single nerve block in which a local anesthetic is injected into the plane between the intercostal muscle and the transverse pectoralis muscle. The initial ultrasound-guided cadaver study found that the TTPB range covered the T2-T6 intercostal nerve [10]. The T2-T6 intercostal nerve innervated the sensory nerve in the medial breast region from the front of the chest, which indicated the TTPB technique has the potential to provide analgesia for anterior chest wall surgery. In subsequent studies, the results of Ueshima et al. also showed that TTPB combined with bilateral thoracic nerve block can successfully complete bilateral breast cancer resection without general anesthesia [11]. The successful use of this technique in mastectomy has paved the way for the use of TTPB in the median sternotomy in cardiovascular surgery. Besides, the rectus sheath nerve block can effectively relieve the pain of the subxiphoid incision and reduce the pain of the incision and drainage orifice[12]. 
First of all, the innervation of the sternum is transmitted by the intercostal nerve originating from the T2T6 thoracic nerve root. The anterior cutaneous branch of the T2-T6 intercostal nerve reaches the lateral edge of the sternum and penetrates subcutaneously. The second anterior branch of the thoracic nerve (T2) distributed in the sternal angle plane, the 6th anterior thoracic nerve (T6) is distributed in the xiphoid plane, innervating the pain receptors of the sternum, transmitting pain caused by sternotomy in heart surgery. So the TTPB range completely covers T2-T6 intercostal nerve anatomically [13]. Secondly, Ueshima et al. reported that the use of bilateral TTPB for patients undergoing cardiac surgery can effectively reduce perioperative pain [14]. There are also current studies showing that TTPB is functioning in breast and heart surgery, cardiac device implantation, pericardiocentesis, and acute and chronic pain management, and without any reports of related major complications so far $[15,16]$. At present, clinical studies have confirmed that reducing the amount of opioids after cardiac thoracotomy and cardiopulmonary bypass can shorten the patient's tracheal tube extubation time and ICU hospital stay [17]. Therefore, the project team speculates that bilateral TTPB can reduce the incision pain of open-heart surgery, reduce the patient's postoperative opioid use, and accelerate the patient's recovery. And the feasibility study published by Fujii et al. [18] showed the potential benefits and feasibility of this technique in cardiac surgery. Considering the pain of drainage orifice, RSB is as a complementary treatment.

However, the TTPB technology was firstly proposed in 2015, and most clinicians do not understand it, and TTPB has a specific application range (it must be done with B-ultrasound), which limits the clinical application of TTPB. In recent years, thanks to the rapid development of nerve block guided by Bultrasound, various nerve block techniques have emerged, making the anesthesia method more accurate, the operation more comfortable, and the postoperative recovery faster. The latest report by Ueshima et al. provides sufficient reference for the method and application of transverse pectoral muscle plane block, including detailed description of puncture method, drug concentration and dosage, and no related serious complications have been seen for the time being. But the lack of long-term continuous observation of pain scores after TTP block surgery, and the lack of record of possible related adverse reactions and patient outcomes, limits the strength of the evidence. Therefore, the project team believes that the design of a randomized double-blind controlled trial can help clarify the application value of TTPB and RSB in the heart surgery under sternotomy.

\section{Methods/design}

\section{Study design}

The protocol of this prospective single-center parallel-group double-blind randomized controlled trial was approved by the institutional review board of the Guangzhou First People's Hospital (K-2021-009-01) and was registered in ClinicalTrials.gov (NCT04838132) before patients were enrolled. This trial is being conducted in Guangzhou First People's Hospital, China. The protocol of this trial conforms to the Standard Protocol Items: Recommendations for Interventional Trials (SPIRIT) guidelines. The schedule of enrollment, intervention, and assessments is based on the SPIRIT diagram. The final report of this trial 
will be written in accordance with the Consolidated Standards of Reporting Trials (CONSORT) statement. The patient flow diagram of the study is presented in Fig. 1, and the study schedule is shown in Table 1(Fig.1 and Table.1).

\section{Informed consent}

Written informed consent will be obtained from each patient before enrollment. They will be informed that they are free to withdraw their consent from the study at any time. The procedure, benefits, risks, and data management of this study will be clarified in detail for the participants during the preoperative conversation.

\section{Participants and recruitment}

Patients undergoing coronary artery bypass grafting (CABG) or heart valve surgery under cardiopulmonary bypass with midsternal incision will be recruited and screened for eligibility. An investigator in this trial will complete the recruitment and sign the informed consent one day before the operation.

Inclusion criteria:

- Patients voluntarily cooperate with the study and sign an informed consent form;

- Ages between 18 and 65;

- American Society of Anesthesiologists (ASA) classification: I-III;

- Undergo coronary artery bypass grafting (CABG) or heart valve surgery with midsternal incision.

Exclusion criteria:

- Refuse to participate in the trial;

- Allergic to the anesthetics or analgesics;

- Suffering with serious systemic diseases (kidney, liver, lungs and endocrine system);

- American Association of Anesthesiologists (ASA) classification: IV-V;

- Unstable hemodynamics;

- History of drug abuse or chronic pain;

- Mental disorders or communication difficulties;

- Died during or immediately after the operation.

\section{Randomization and Blinding}

Following recruitment, the participants will be randomly allocated to one of the two groups: (1)TTPB+RSB group: bilateral TTPB and RSB with ropivacaine and (2)Control group: bilateral transverse pectoralis plane and rectus sheath puncture with placebo-saline. They will be allocated in a 1:1 ratio. The enrolled patient will have a random number (as their identification code) generated by Microsoft Excel 2016 
(Microsoft Corp., Redmond, WA, United States) [19]. The random number sequence of the patients is enclosed in a sealed envelope, and only one number is written on the outside of each envelope. When a patient is enrolled, a designated intervener will open the envelope and divide it into the control group or test group according to the principle of randomization.

After the recruitment, the informed investigator will open the envelope with the same serial number to determine the corresponding intervention plan for patient. The supervising physician, the anesthesiologist of the operation, and the postoperative follow-up doctor are all unaware of the grouping. After the completion of all inclusion and data collection, the blind will be uncovered, and the corresponding number of subjects is taken out to obtain grouping information. During the entire trial period, the investigator is not allowed to uncover the blind unless there is a necessary medical reason. In the event of emergency, such as a serious adverse event, the investigator can immediately unblind the subject in order to understand the medication status of the subject, so as to ensure the correct medical treatment for the patient. Once the blindness is uncovered, the subject will immediately withdraw from the study.

\section{Intervention}

\section{Management of general anesthesia}

On the day of surgery, vital signs were monitored by the American Society of Anesthesiologists upon admission to the operating room.

General anesthesia induction: midazolam $0.5 \mathrm{mg} / \mathrm{kg}$, remifentanil $1 \mathrm{ug} / \mathrm{kg}$, propofol $2 \mathrm{mg} / \mathrm{kg}$, and rocuronium $0.9 \mathrm{mg} / \mathrm{kg}$. After smooth induction, the endotracheal catheter was inserted smoothly.

Anesthesia maintenance: After tracheal intubation, the maintenance medication is $1-2 \%$ sevoflurane, propofol $2-5 \mathrm{mg} / \mathrm{kg} / \mathrm{h}$, intermittent bolus injection of rocuronium. Pump injection of remifentanil 0.1-0.4 $\mathrm{ug} / \mathrm{kg} / \mathrm{min}$ before and after cardiopulmonary bypass, remifentanil $1 \mathrm{ug} / \mathrm{kg} / \mathrm{min}$ is pumped during extracorporeal circulation [20] (injection can be reduced or suspended according to clinical vital signs). The adjustment of the drug dosage was adjusted by cardiovascular anesthesiologists who were ignorant of this trial design group according to the patient's vital signs and surgical progress, maintaining the depth of anesthesia Bispectral Index (BIS) between 40-60, and the mean arterial pressure fluctuation between $60-80 \mathrm{mmHg}$.

Postoperative analgesia: All patients were given hydromorphone $0.5-2 \mathrm{mg}$. iv and ondansetron $4 \mathrm{mg}$.iv during the sternum suture with steel wire. A 48-hour intravenous self-control analgesic pump was used after the operation. Analgesic formula: Sufentanil 150ug+ Butorphanol tartrate 6mg+ Dexmedetomidine $200 \mathrm{ug}+$ Tropisetron $10 \mathrm{mg}+0.9 \% \mathrm{NS}$, a total volume of $150 \mathrm{ml}$, the load volume is $3 \mathrm{ml}$, the continuous volume: $2 \mathrm{ml}$, the single volume is $2 \mathrm{ml}$, the lock time is $30 \mathrm{~min}$, and the limit is $10 \mathrm{ml}$ per hour.

At the end of the operation, if the patient meets the extubation criteria, try extubation in the operating room; or infused with propofol and transferred to the ICU. 
Remedial medication for postoperative pain: when the patient's VAS score $\geq 4$, intravenous tramadol 1 $\mathrm{mg} / \mathrm{kg}[21]$.

\section{Ultrasound-guided transversus thoracis muscle plane block and rectus sheath block}

The technique of TTPB was described by Ueshima. After the patient's tracheal intubation, an experienced anesthesiologist who was unaware of the grouping of the patients underwent ultrasound-guided bilateral TTP block. A linear array transducer $(6-13 \mathrm{MHz})$ with a sterile cover and a 22-gauge (G) block needle $\left(\mathrm{KDL}^{\mathrm{T}}{ }^{\mathrm{M}}\right.$, Kindly group, Shanghai, China) will be used. Place the ultrasound probe on the longitudinal plane $1 \mathrm{~cm}$ outside the edge of the sternum in the fourth intercostal space, and identify the T4-T5 intercostal space under ultrasound (Figure 2(A)). On the pleura, the internal thoracic intercostal muscles, transverse pectoralis muscles, internal chest arteries and veins can be seen(Figure 2(B))[15]. Above the pleura is the parasternal sagittal plane, and the intercostal muscles and transversus pectoralis between the fourth and fifth ribs can be seen. Use the plane technique to place a 22-gauge, $80-\mathrm{mm}$ needle obliquely upwards until the needle tip is located at the plane between the internal intercostal muscles and the transverse pectoralis muscle (Figure 2(C)). Observation of the pleura downward movement by injecting $3 \mathrm{~mL}$ of normal saline confirms the right position of the needle tip [18]. After excluding the needle tip in the blood vessel and the pleura, $20 \mathrm{ml}$ of $0.3 \%$ (body weight $<75 \mathrm{~kg}$ ) or $0.5 \%$ ropivacaine (body weight $\geq 75 \mathrm{~kg}$ ) were given on both sides, respectively. In addition, place the ultrasound probe on the longitudinal plane $3 \mathrm{~cm}$ below the edge of the xiphoid in the Hunter's line, and identify the rectus under ultrasound (Figure 2(D)and (E)). Use the plane technique to place a 22-gauge, $80-\mathrm{mm}$ needle obliquely upwards until the needle tip is located at the posterior sheath of rectus abdominis, $10 \mathrm{ml}$ of $0.3 \%$ (body weight $<75 \mathrm{~kg}$ ) or $0.5 \%$ ropivacaine (body weight $\geq 75 \mathrm{~kg}$ ) were given on both sides, respectively.

The control group used the same volume of normal saline instead of local anesthetics with the same puncture process.

\section{Intraoperative monitoring and management}

The patient's heart rate $(\mathrm{HR})$, invasive arterial blood pressure (IBP), pulse oxygen saturation $\left(\mathrm{SpO}_{2}\right)$, endtidal carbon dioxide (PetCO2), central venous pressure (CVP), and body temperature were monitored throughout the operation. Intraoperative mean arterial pressure (MAP) 30\% higher (or lower) than the baseline value will be defined as hypertension (or hypotension). For hypotension, intravenous ephedrine 5-10 mg or phenylephrine 50-100 ug will be used in time, and urapidil 5-10 mg will be used for hypertension. Bradycardia (defined as a heart rate less than 60 beats/minute) will be given intravenous injection of $0.5 \mathrm{mg}$ of atropine. The goal of intraoperative anesthesia management is to maintain hemodynamic stability before cardiopulmonary bypass (heart rate $<100$ beats $/ \mathrm{min}, \mathrm{MAP}>60 \mathrm{~mm} \mathrm{Hg}, \mathrm{CVP}$ $8-12 \mathrm{mmHg}$, urine output $>0.5 \mathrm{ml} / \mathrm{kg} / \mathrm{h}$ and mixed venous oxygen saturation $>70 \%$ ). Pump dobutamine or epinephrine after the bypass, maintaining HR>90, SBP $>100$. When the MAP is lower than the target value during the operation, treatment programs such as fluid replacement and vasoactive drugs are adopted according to the patient's condition. 


\section{Outcome Definitions}

\section{Primary outcome evaluation}

A. Incidence of hyperalgesia: 48 hours after the operation, a $180 \mathrm{mN}$ von-Frey wire (Touch Test ${ }^{\mathrm{TM}}$ Sensory Evaluator, USA) was used to test the pain sensitivity of the area around the wound as described in the literature [22] to evaluate the hyperalgesia. On the skin around the incision, the first point where the patient experiences a "sharp" to "severe pain" sensation is marked, and the distance to the incision is recorded. The data will be standardized to exclude the effect of incision length on the hyperalgesic area.

B. The total amount of analgesics used in the perioperative period.

During the perioperation, the amount of analgesics during the operation, the total amount of analgesic pump drugs used and the frequency of pressing the additional bolus trigger in the patient's analgesia system at 48 hours after the operation, and the amount of remedial analgesics are recorded. An additional dose of intravenous tramadol $(1 \mathrm{mg} / \mathrm{kg}$ ) was added when the VAS score was still $\geq 4$ points after the patient triggered the additional dose of the analgesic pump. (Intravenous self-control analgesia pump is an intravenous fluid infusion device that gives a continuous infusion volume to maintain a stable concentration of the drug in the blood. In addition, the patient can press it by itself to increase an additional infusion dose for a limited time Only one effective press.)

\section{Secondary outcome evaluations}

- Pain score after operation (VAS score of patients at $0,6,12,24,48 \mathrm{~h}$ after extubation)

- Time of extubation (time from the end of the operation to the tracheal tube removal)

- Length of ICU stay (the times of stay in ICU after operation)

- Time to ground activity (the number of days the patient can get out of bed after the operation)

- Length of stay in hospital.

- Postoperative complications (such as nausea and vomiting, pulmonary infection, wound infection, delayed healing, renal insufficiency, etc.)

- Complications related to anesthesia (local anesthetic systemic toxicity, pneumothorax, epidural block, total spinal block, hematoma, etc.)

\section{Sample size calculation}

The Gpower3.1.9.7 software was used to estimate the sample size, according to the previous study that the mechanical pain threshold (primary endpoint) of the control group at $48 \mathrm{~h}$ after surgery was $1.2 \pm 0.56$ $[\log (\mathrm{g})][22]$. Hypothesis the incision pain threshold at $48 \mathrm{~h}$ after the TTP treatment could be increased by $33 \%$ to $1.6 \pm 0.56[\log (\mathrm{g})]$ (the normal mechanical pain threshold is $1.99 \pm 0.24[\log (\mathrm{g})])$. The calculated effect size of two independent sample mean $t$ test method is 0.71 . A sample size of 35 for each group will achieve $90 \%$ power to detect the difference with a two-tailed $5 \%$ significance level. In order to increase 
the reproducibility of the study and avoid incomplete data collection and loss to follow-up, 80 patients are planned to be included.

\section{Statistical analysis}

SPSS V.24.0 (IBM Corporation, Armonk, New York, USA) will be used for statistical analysis. The Kolmogorov-Smirnov test is used to evaluate the normal distribution of the data. Descriptive statistics include the average value of the standard deviation or proportion according to the characteristics of the data. Numerical variables are represented by the mean \pm standard deviation (SD) or median (interquartile range). The comparison of independent variables between groups will be analyzed by Student's unpaired $t$ test, whereas non-parametric data will be compared using the Mann-Whitney $U$ test. Categorical variables will be expressed as frequency (\%). A chi-square test or Fisher's exact test will be used for categorical variables. A two-tailed, $\mathrm{P}<0.05$ will be considered statistically significant.

\section{Data collection, monitoring and management}

Preoperative, intraoperative and 48h postoperative follow-up data will be collected from electronic medical records, nursing record, monitoring machines and relevant manual records by the case report form (CRF). Data collection and data quality control are the responsibility of the researcher and the principal responsible person respectively.

\section{Harms}

In the event of an unpredicted adverse event, the operator should immediately notify the superior doctor and deal with it according to clinical guideline. All adverse events that occurred will be recorded on the "Adverse Event Record" page of the CRF form, and the treatment and outcome also will be recorded in detail. Serious adverse events should be recorded in the "Serious Adverse Event Record Form" and reported to the Ethics Committee of Guangzhou First People's Hospital within 24 hours.

\section{Auditing}

No formal auditing process is proposed for this trial.

\section{Termination criteria}

- The occurrence of "unpredictable" serious adverse events, or the incidence of adverse reactions and conditions reported in domestic and foreign research journals both indicate that it is not appropriate to continue the test;

- The latest research reports indicate that it may cause cancer, other major diseases, injuries or deaths;

- Once all the blinds are leaked, or the unblinding rate exceeds $20 \%$, it means that the double-blind trial has failed, and this clinical trial needs to be terminated.

\section{Data retention}


The investigator will take out the CRF form from the main investigator at an appropriate time for followup and data collection of the subjects. In strict accordance with the clinical research plan, fill in the form according to the guidelines and content of the form. After completing the form, the CRF form should be returned immediately and the main investigator stored in a safe with moisture and fireproof conditions. Investigators shall observe confidentiality obligations and shall not copy or disseminate privacy and CRF forms of patients.

\section{Protocol amendments}

Any change in the study protocol will require an amendment. Any proposed protocol amendments will be initiated by the principal investigators. All amended versions of the protocol will be signed by the staff in the study and the amendment forms will be submitted to the Ethics Committee for approval.

\section{Trial dissemination}

The outcomes of the study will be disseminated in a peer-reviewed journal or at scientific conferences.

\section{Discussion}

The TTP block is a familiar technology benefitting from the development of the ultrasound and the application of nerve blocking. In the other words, the TTP block on transthoracic median incision heart surgery just a ramification of nerve block anesthesia. Therefore, the prevalence of TTP block will be coming. But this skill lacks the evidence-based medical evidence and clinical data support at present, which is the original intention of the experiment.

Thanks to the visualization development of ultrasound, the safety factor of TTP block is greatly improved and the block effect is more accurate, which provides a guarantee for the application of TTP. The purpose of this study was to explore the application value of TTP block in midsternal incision cardiac surgery, including reducing perioperative opioid dosage and postoperative hyperalgesia, shorting ventilator ventilation time and ICU hospitalization days, so as to make patients recover quickly and reduce the treatment cost of patients.

The study is only a single-center study and may not be applicable to other units or different types of patients. But the results of this study may lead to the development of new models of perioperative cardiac analgesia and the optimal management of pain during cardiac surgery.

\section{Trial status}

The trial is currently in recruitment phase. Trial completion is expected by December 2021.

\section{Abbreviations}

TTPB: Transversus Thoracis muscle Plane Block 
RSB: Rectus Sheath Block

VAS: Visual Analogue Scale

ERAS: Enhanced Recovery After Surgery

CABG: Coronary Artery Bypass Grafting

ASA: American Society of Anesthesiologists

BIS: Bispectral Index

HR: Heart Rate

IBP: Invasive Arterial Blood Pressure

$\mathrm{SpO}_{2}$ : Pulse Oxygen Saturation

PetCO $_{2}$ : End-tidal Carbon Dioxide

CVP: Central Venous Pressure

MAP: Mean Arterial Pressure

SBP: Systolic Blood Pressure

CRF: Case Report Form

\section{Declarations}

\section{Ethics approval and consent to participate}

This trial was approved supervised by the institutional review board of the Guangzhou First People's Hospital (K-2021-009-01) and was registered in ClinicalTrials.gov (NCT04838132) before patients were enrolled. Written, informed consent to participate will be obtained from all participants.

\section{Consent for publication}

These are available from the corresponding author on reasonable request.

\section{Availability of data and materials}

The material of this study will be conserved in a secure repository at the Anesthesiology Department, Guangzhou First People's Hospital, South China University of Technology. Datasets will be available from the $\mathrm{PI}$ upon reasonable request. 


\section{Competing interests}

The authors declare that they have no competing interests.

\section{Funding}

This trial is supported by the Health Science and Technology Project of Guangzhou City (20211A010007).

\section{Authors' contributions}

Bin Zheng and Lixin Xu are the Chief Investigator, they conceived the study, led the proposal and protocol development. Jierong Luo and Chengxiang Lu contributed to study design and to development of the proposal. Haitao Zhou and Guokun Ou were the lead trial methodologist. All authors read and approved the final manuscript.

\section{References}

1. Shengshou Hu. G.R., LIU and Z.m. lisheng, WAN wen,wang yongjun, wu zhaosu, li hujin, gu dongfeng., Summary of the 2018 Report on Cardiovascular Diseases in China. Chinese Circulation Joural. 2019;34(3):209.

2. Cogan J. Pain management after cardiac surgery. Semin Cardiothorac Vasc Anesth. 2010;14(3):201-4.

3. Perkins FM, Kehlet H. Chronic Pain as an Outcome of Surgery. Anesthesiology. 2000;93(4):1123-33.

4. Bignami E, et al., Perioperative pain management in cardiac surgery: A systematic review. Minerva Anestesiologica, 2017. 84(4).

5. Kehlet $\mathrm{H}$, Holte K. Effect of postoperative analgesia on surgical outcome. Br J Anaesth. 2001;87(1):62-72.

6. Fletcher D, Martinez V. Opioid-induced hyperalgesia in patients after surgery: a systematic review and a meta-analysis. $\mathrm{Br} \mathrm{J}$ Anaesth, 2014(6): p. 991-1004.

7. Noba L, et al. Enhanced Recovery After Surgery (ERAS) Reduces Hospital Costs and Improve Clinical Outcomes in Liver Surgery: a Systematic Review and Meta-Analysis. J Gastrointest Surg. 2020;24(4):918-32.

8. Martucci KT, et al. Opioid-independent mechanisms supporting offset analgesia and temporal sharpening of nociceptive information. Pain. 2012;153(6):1232-43.

9. Ueshima H, Kitamura A. Blocking of Multiple Anterior Branches of Intercostal Nerves (Th2-6) Using a Transversus Thoracic Muscle Plane Block. Regional Anesthesia \& Pain Medicine, 2015. 40(4): p. 388.

10. Ueshima H, Ishikawa YT,S, Otake H. Ultrasound-guided transversus thoracic muscle plane block: a cadaveric study of the spread of injectate. J Clin Anesth. 2015;27(8):696-6. 
11. Ueshima H, Kitamura A. Clinical experiences of ultrasound-guided transversus thoracic muscle plane block: a clinical experience. Journal of Clinical Anesthesia, 2015.

12. Landmann A, Visoiu M, Malek MM. Development of a novel technique for bilateral rectus sheath nerve blocks under laparoscopic-guidance. J Pediatr Surg. 2017;52(6):966-9.

13. Goldwyn RM. Gray's anatomy. Plast Reconstr Surg. 1985;76(1):147-8.

14. Marui, et al., The ultrasound-guided transversus thoracic muscle plane block is effective for the median sternotomy. Journal of Clinical Anesthesia, 2016.

15. Fujii S, et al. Transversus Thoracis Muscle Plane Block. Biomed Res Int. 2019;2019(4):1-6.

16. Ueshima $\mathrm{H}$, Otake $\mathrm{H}$. Addition of transversus thoracic muscle plane block to pectoral nerves block provides more effective perioperative pain relief than pectoral nerves block alone for breast cancer surgery. Br J Anaesth. 2017;118(3):439-43.

17. PG G, et al. Effect of opioid-free anaesthesia on post-operative period in cardiac surgery: a retrospective matched case-control study. BMC anesthesiology. 2019;19(1):136.

18. Fujii S, et al. Transversus thoracis muscle plane block in cardiac surgery: a pilot feasibility study. Reg Anesth Pain Med. 2019;44(5):556-60.

19. Kim J, Shin W. How to do random allocation (randomization). Clin Orthop Surg. 2014;6(1):103-9.

20. Krishna SN, et al. Bilateral Erector Spinae Plane Block for Acute Post-Surgical Pain in Adult Cardiac Surgical Patients: A Randomized Controlled Trial. J Cardiothorac Vasc Anesth. 2019;33(2):368-75.

21. Subramaniam K, et al., Remifentanil and perioperative glycaemic response in cardiac surgery: an open-label randomised trial. British Journal of Anaesthesia, 2020.

22. Bornemann-Cimenti $\mathrm{H}$, et al. Preoperative pregabalin administration significantly reduces postoperative opioid consumption and mechanical hyperalgesia after transperitoneal nephrectomy. BJA: British Journal of Anaesthesia. 2012;108(5):845-9.

\section{Table}

Due to technical limitations, table 1 is only available as a download in the Supplemental Files section.

\section{Figures}




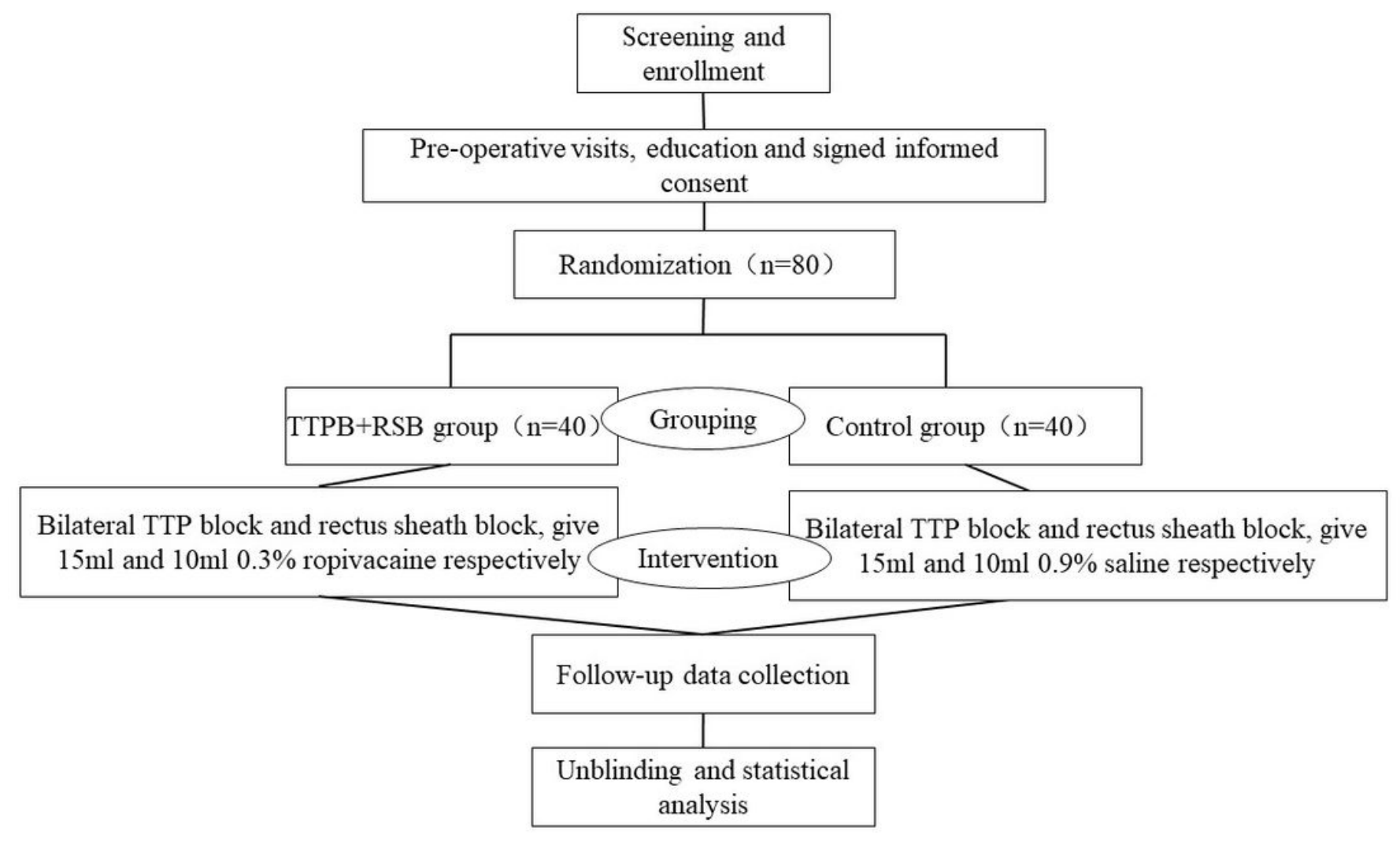

\section{Figure 1}

Consolidated standards of reporting trials (CONSORT) flow diagram. TTPB+RSB group: bilateral transverse thoracic muscle plane block and rectus sheath block, give $15 \mathrm{ml}$ and $10 \mathrm{ml} 0.3 \%$ ropivacaine respectively; Control group: bilateral TTP block and rectus sheath block, give $15 \mathrm{ml}$ and $10 \mathrm{ml} 0.9 \%$ saline respectively. 

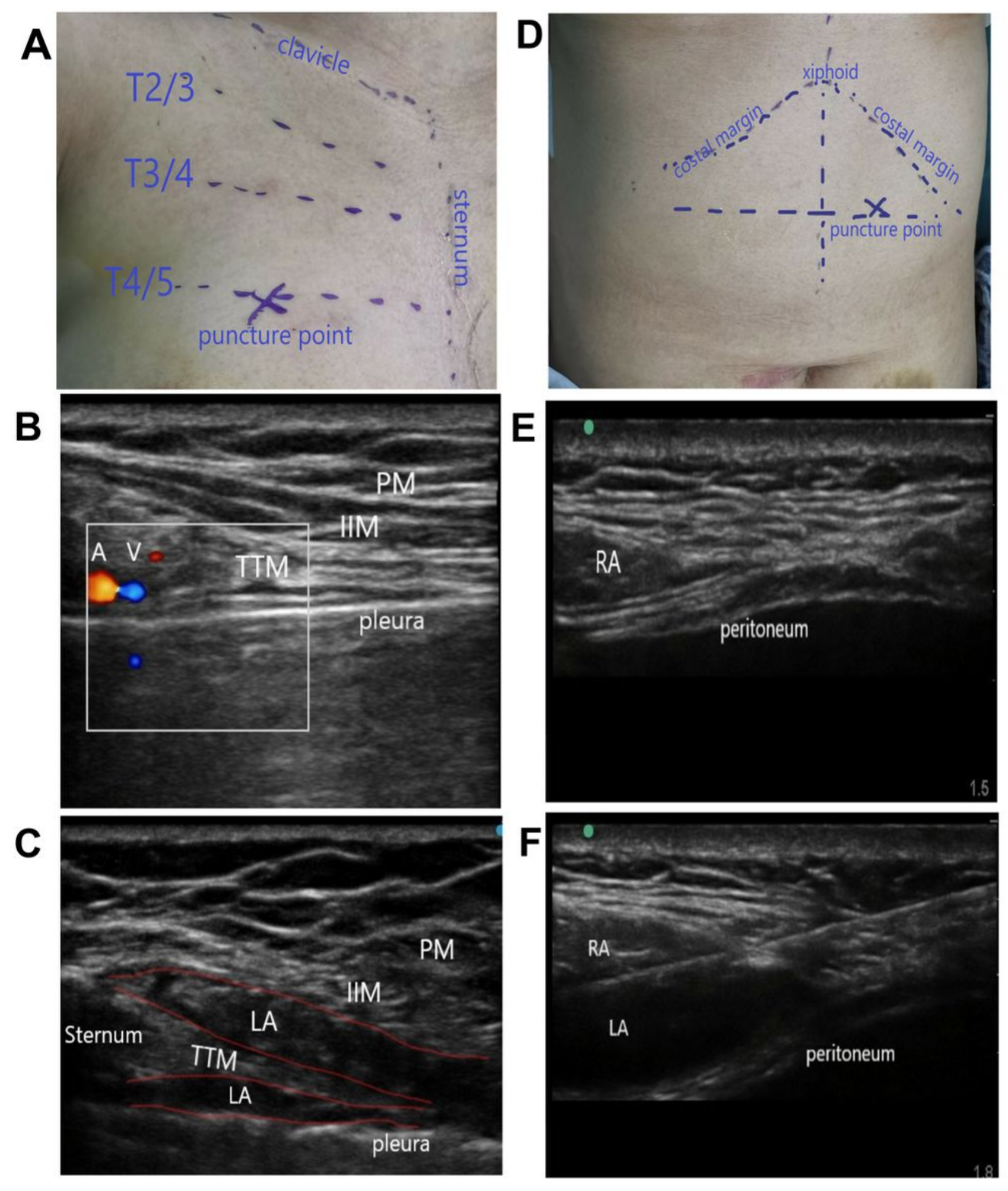

Figure 2

(A) Body surface location of puncture in transverse pectoralis plane block (TTP). (B) Ultrasound-image of $T 4 / 5$ intercostal cross-section of the transverse thoracic muscle plane. (C) Ultrasound-image of local anesthetic (LA) diffusion on the transverse thoracic muscle plane. LA surrounds the transverse thoracic muscle (TTM), the internal thoracic artery(A), and the internal thoracic vein(V). (D) Body surface location of puncture in rectus sheath block (RSB). (E) Ultrasound-image of transverse section of the rectus 
abdominis (RA) below the xiphoid process. (F) Ultrasound-image of LA diffusion on the posterior sheath of the rectus abdominis. PM: Pectoralis Muscle; IIM: Internal Intercostal Muscle.

\section{Supplementary Files}

This is a list of supplementary files associated with this preprint. Click to download.

- Table1.xlsx

- SPIRITchecklist.docx 